\title{
Evaluation of Different Combinations of Gated Trapping, RF-Only Mode and Trap Compensation for In-Field MALDI Fourier Transform Mass Spectrometry
}

\author{
Jonathon K. Gooden, Don L. Rempel, and Michael L. Gross \\ Department of Chemistry, Washington University, St. Louis, Missouri, USA
}

\begin{abstract}
MALDI, while providing advantages such as the ability to do in-depth and repeated exploration of the sample, challenges the existing performance capabilities of Fourier transform mass spectrometry (FTMS). The challenge arises because MALDI-produced ions have high mass-to-charge ratios and uncertain kinetic-energy distributions. We demonstrate that a combination of a gated trapping event, a RF-only mode pressure focusing event, and an electrically compensated trap provides a compelling advantage in meeting these challenges. Removal of any of the above combination elements significantly degrades the detection performance of substance $\mathrm{P}$ from $850 \mathrm{~K}$ resolving power at $34.9 \mathrm{kHz}$ and of melittin from 278 $\mathrm{K}$ resolving power at $16.5 \mathrm{kHz}$ when using a 3-Tesla magnet-based spectrometer. (J Am Soc Mass Spectrom 2004, 15, 1109-1115) (C) 2004 American Society for Mass Spectrometry
\end{abstract}

$\mathrm{M}$ atrix-assisted laser desorption ionization (MALDI), when interfaced to Fourier transform mass spectrometry (FTMS) in principle has advantages with respect to electrospray ionization (ESI). First, MALDI places a lower vacuum load than does ESI on the instrument, permitting a simplified high vacuum system. Second, the low charge-state ions that are produced by MALDI allow more ions in the trap for a given charge capacity and give better utilization of the limited dynamic range of FTMS. Third, when the sample probe is located at the trap, a simple instrument results, negating the use of an external ion source, transmission ion optics, and associated pumping. The combination of random access and retrieval, a general characteristic of MALDI, and the versatility of FTMS (e.g., accurate mass, ion manipulation and activation) facilitate in-depth and repeated exploration of unknown samples.

The shortcomings of FTMS, however, are more apparent with MALDI-produced than ESI-produced ions. The principal problem is that the former ions have higher $m / z$, lower frequency than do corresponding ESI ions. The former ions often have a lower charge state, causing poor performance because magnetic forces decrease as $\mathrm{m} / \mathrm{z}$ increases. Use of higher B fields reduces the problem while shifting it to higher $m / z$. Further-

Published online June 9, 2004

Address reprint requests to Michael L. Gross, Department of Chemistry, Washington University, P.O. Box 1134, One Brookings Drive, St. Louis, Missouri, USA. E-mail: mgross@wustl.edu more, distributions of initially formed MALDI ions are variable and broad with high velocities (up to $\sim 660$ $\mathrm{m} / \mathrm{s}$ ) [1], resulting in inefficient "catching" and unreliable and often poor detection.

An effective and logical plan to improve MALDI/ FTMS could include three general approaches. The first is to modify the MALDI desorption to attenuate the translational velocities and internal energies of the desorbed ions. Examples of these efforts include the use of a comatrix [2] or a "waiting room," [3] which in the limit becomes high-pressure MALDI [4]. An alternative is the use of an external source [5], which permits the use of quadrupole and higher order traps or ion guides to focus and thermalize the MALDI-produced ions before introducing them into the FTMS trap. The second approach is to introduce efficiently the MALDI ions, whether produced externally or in-field, into the trap. The third is to improve the trap for detection of the high- $m / z$ ions that are often encountered in the MALDI experiment. We suggest that correcting the trapping electric fields is essential for this approach. In this article, we describe improvements found when adaptations from the second and third areas are incorporated into an in-field MALDI experiment. Before describing our results, we briefly review the precedents for the three chosen strategies that we use in combination.

Gated trapping is a common method for catching in-field MALDI-generated ions. Two efforts to improve performance through changes in gated trapping include shaping the trap electric field [6] and exploiting cylindrical traps for improved mass range [7]. A third 
approach involves trapping ions with the wide velocity range by using parabolic retarding potentials [8], large retarding potentials [9], a theoretical "two-time constant and a delay" gated trapping strategy [10], and smooth deceleration at the entrance of an open cylindrical trap [11].

Early examples of correcting electric fields (compensation) in Penning traps were by Van Dyck et al. [12, 13] who used traps of hyperbolic geometry in physics experiments. Gabrielse later compensated a cylindrical Penning trap, and analyzed the cylindrical [14] and hyperbolic geometries [15] based on polynomial expansions of the potential at the trap origin. Subsequently Inoue et al. [16, 17] compensated a cylindrical trap for application in FTMS by first considering concentrically divided trapping electrodes and then building a trap with three pairs of ring electrodes arranged along the trap axis with the goal of reducing the radial electric field. Later, Marshall and co-workers [18] constructed a matrix-shimmed trap in which all electrodes of the trap were each segmented into 25 squares to effect control of the electric field shapes to obtain a more globally quadrupolar trapping potential.

Correcting the electric field for trapping must be accompanied by a method to reduce and center the ion cloud, which is especially diffuse when ions are made in-field. One means for this is quadrupolar excitation, first introduced to mass spectrometry by Schweikhard et al. $[19,20]$, and further developed by Marshall and co-workers [21, 22]. Quadrupolar excitation effects the ion-cloud size reduction by converting magnetron into cyclotron motion, which is then collisionally damped. Another method to reduce ion-cloud size is to add the features of the Paul trap to the Penning trap. The former trap, because it operates at 4-5 orders of magnitude higher pressure than the latter, has the ability to pressure focus. Paul and Penning traps were first combined by Fisher [23] and later discussed by Li and coworkers $[24,25,26]$. Gorshkov et al. [27] implemented a lowvoltage, low-frequency version of the combined trap for simultaneous trapping and detection of positive and negative ions in FTMS.

We extracted from these strategies three independent approaches for improving instrument performance: gated trapping with a shaped retarding field, electrical compensation with two-segment trapping electrodes [28, 29, 30, 31, 32], and an RF-only-mode event $[33,34,35]$. The utility of combining the RF-onlymode event and compensation was first demonstrated by using an 1.2-T FT instrument: ions from a test system (benzene) were distributed in the trap by excitation of the magnetron mode to a diameter that is half the size of the cubic trap to emulate the outer reaches of an ion distribution that might be produced by MALDI. Without compensation or RF-only-mode, no signal was observed. We found a low resolving power signal with just the RF-only mode operating, a weak, but highly resolved signal with compensation, and finally, an intense high resolving-power signal with both the RF- only-mode event and compensation turned on [36]. We reproduced the trend with $\mathrm{C}_{60}$ evaporated from a heated solids probe [37]. In a larger $4.76-\mathrm{cm}$ trap in a higher $3.0 \mathrm{~T}$ magnetic field, the resolving power not only improved from 25,000 to $1,000,000$ via compensation of laser-desorbed $\mathrm{C}_{60}$, but could be sustained from shot to shot with a precision of $\pm 19 \%$ [38].

Subsequently, we showed that the $[\mathrm{M}+\mathrm{H}]^{+}$of substance $\mathrm{P}(\mathrm{m} / \mathrm{z}$ 1347.7, $\mathrm{f} \sim 36 \mathrm{kHz})$, which was introduced by MALDI, could be detected with a resolving power of 310,000 in a compensated trap with an ion cloud sized by quadrupolar excitation whereas we found only lower resolving power signals in the absence of compensation, with or without quadrupolar excitation [39]. We then demonstrated that the RF-onlymode event is a suitable replacement for quadrupolar excitation [40].

\section{Experimental}

The peptides substance $\mathrm{P}$ and melittin were purchased from Sigma (St. Louis, MO) and used without further purification. Samples were dissolved in 1:1 MeOH/ $\mathrm{H}_{2} \mathrm{O}$ at concentrations of approximately $20 \mathrm{pmol} / \mu \mathrm{L}$. The matrix, 4-OH- $\alpha$-cyanocinammic acid (CHCA) (Sigma), was prepared as a saturated solution in 1:1 $\mathrm{AcN} / \mathrm{MeOH}$. Although not the first choice for FTMS experiments, CHCA was chosen as a representative of a worst case scenario and, if CHCA should work, other matrices should as well.

Ions were generated by MALDI using the third harmonic (355 nm) from a Nd:YAG laser (Quanta-Ray DCR-2(10)) aimed along the trap z-axis and located opposite the probe positioned $4 \mathrm{~mm}$ from the trap. Ion production was adjusted by use of a circular variable attenuator (Newport optics model 50GOOAV.1). The laser power was reduced to a point just above the detection threshold for the signal of the ions of interest. A MIDAS data system with a Hewlett Packard (Everett, Washington) A-to-D converter (model HPE1437A) and a National Instruments (Austin, Texas) arbitrary waveform generator (model NI5411) was used to control the experiment.

To achieve asymptotic stability over a wide mass range (100 to 75,000 m/z) [39] during the RF-only-mode event, a $4.8-\mathrm{kV}$ base-to-peak $0.885-\mathrm{MHz}$ sine wave would be required. A waveform approximating this (3.3-kV base-to-peak $0.90485-\mathrm{MHz}$ sine wave) was generated with a coil that resonates with the network of stray capacities of the trap and its leads. The RF-onlymode event was typically operated while He gas was introduced $\left(4 \times 10^{-5}\right.$ torr for substance $\mathrm{P}$ and $9 \times 10^{-5}$ torr for melittin experiments), and the RF was continued (stretch time) while the system was re-evacuated. Experiments used a 40-s RF/He time and a 20-s stretch time for substance $\mathrm{P}$ and a 1-s RF/He time and a 2-s stretch time for melittin.

To effect the RF-only mode in the FT trap, the tuned radio coil and MOSFET switch array, at the base of the 


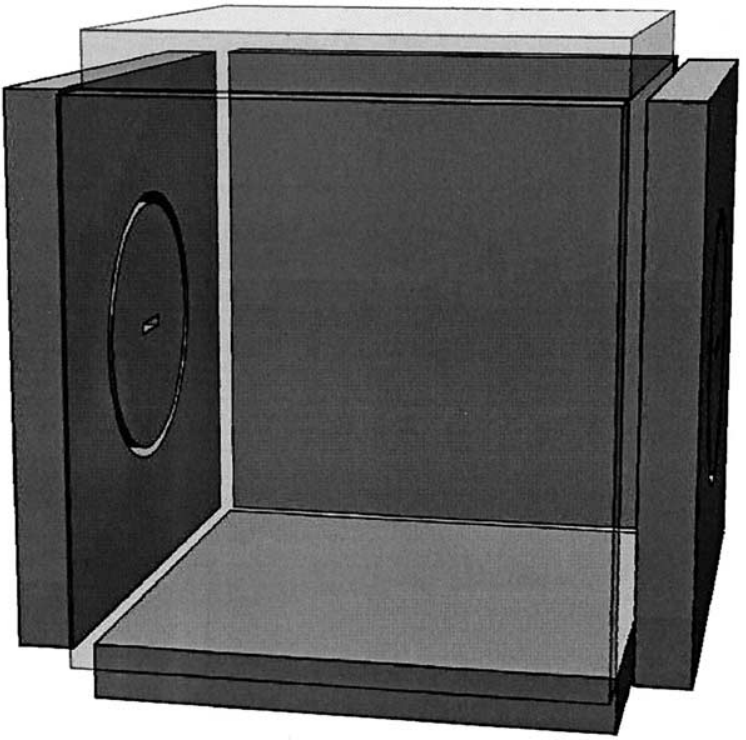

Figure 1. Compensated cubic trap showing segmented trapping plates consisting of a center disk and outer segments. For detection, the center disk was at $0.50 \mathrm{~V}$ and the outer segment at $0.85 \mathrm{~V}$ and $0.89 \mathrm{~V}$ for substance $\mathrm{P}$ and melittin, respectively.

coil, were used to intercept the analog drives normally connected to the trapping plates of the existing configuration of a 3-Tesla Finnigan T30 FTMS mass spectrometer [41]. The switch array permitted the rapid switching of the trapping voltages supplied through the coil to the trapping plates. The two $47.6-\mathrm{mm}$ cubic trapping plates were previously segmented into an electrically independent, 23.8-mm center disk and an outer segment (see Figure 1), permitting electrical compensation of the trapping electric field during detection. The trap segment voltages were $0.85 \mathrm{~V}$ for substance $\mathrm{P}$ and 0.89 $\mathrm{V}$ for melittin applied to the outer segments and $0.5 \mathrm{~V}$ applied to the disks during excitation and detection. The compensated trapping voltages were optimized for each protein. The values were experimentally different from one another, and we conclude from this that there is a mass dependence.

Different voltages supplied to the segments during the gated-trapping events shaped the retarding electric field used to catch the ions. For experiments that involved substance $\mathrm{P}$, the voltages used during the gated-trapping event were $-30.0 /-10.0 \mathrm{~V}$, for the entrance trapping plate outer segment/disk, and 0.0/0.0 $\mathrm{V}$ for the exit trapping plate outer segment/disk. The potential surface created in the trap for this example is shown in Figure 2. Unlike the surface used by Wilkins and co-workers [6], which was the first shaped retarding field used in FTMS, the surface in Figure 2 requires the higher energy ions that penetrate the furthest into the trap do work in the radial direction as they are decelerated in the $\mathrm{z}$ direction whereas this is not the case for the retarding field implemented by Wilkins. The voltages used were held for approximately $60 \mu \mathrm{s}$ after the laser shot. No effort was made to optimize

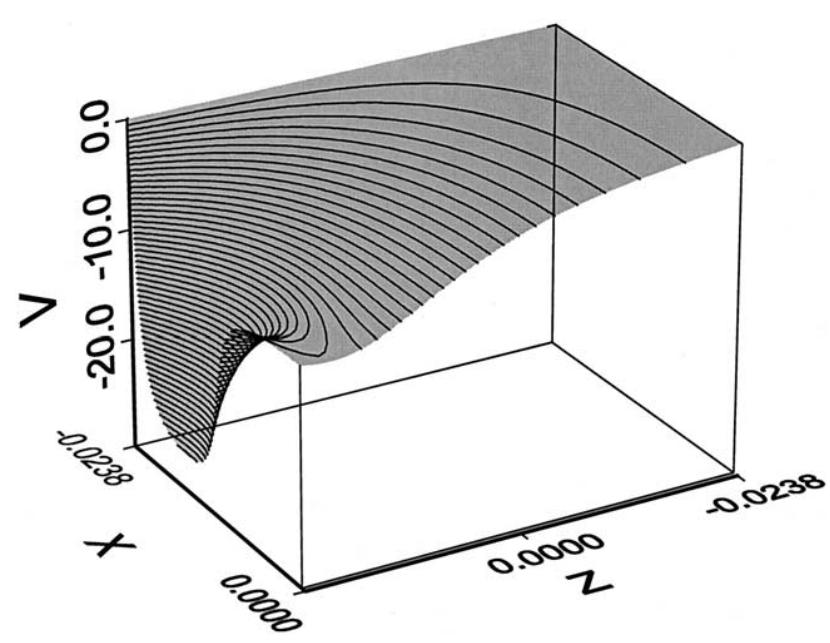

Figure 2. Electrical potential surface applied to retard the $(\mathrm{M}+$ $\mathrm{H})^{+}$ions of substance $\mathrm{P}$ during the first $63.6 \mu$ s after the laser desorption event. A similar surface (reduced by a factor of 0.6 ) was employed for retarding the $(\mathrm{M}+\mathrm{H})^{+}$ions of melittin.

carefully the voltages or the time for the gated trapping event in the experiment sequence for substance P. For experiments that involved melittin, the voltages used during the gated-trapping event were $-18.0 /-6.0 \mathrm{~V}$, for the entrance trapping plate outer segment/disk, and $0.0 / 0.0 \mathrm{~V}$ for the exit trapping plate outer segment/disk (the resulting surface has the same overall shape as that shown in Figure 2 but its depth is more shallow). These voltages were also held for approximately $60 \mu \mathrm{s}$ after the laser shot. The gated trapping voltages $-18.0 /-6.0$ gave better performance when compared to $-30.0 /$ -10.0 for melittin, but these voltages were not carefully optimized. The electrically isolated probe tip was electrically connected the adjacent trapping plate disk.

In order to avoid limiting the measured resolving power by an optimal ( 3 times the transient decay time constant) detection time, an excessively long detection time was used to show better peak shape detail at the expense of a better signal-to-noise ratio. The data acquisition time was 104.9 seconds for all experiments during which $4-\mathrm{M}$ sample values were acquired with a $10-\mathrm{kHz}$ band width and center frequencies of $34.8-\mathrm{kHz}$ for substance $\mathrm{P}$ and $16.2-\mathrm{kHz}$ for melittin. No apodization was used in these experiments. All spectra show the same frequency width of $6.08 \mathrm{~Hz}$. One minor tick interval is approximately $0.16 \mathrm{~Hz}$. Resolving powers were computed graphically based on the full peak width at half maximum (FWHM).

\section{Results and Discussion}

The earlier results for $\mathrm{C}_{60}$ at $1.2 \mathrm{~T}$ and substance $\mathrm{P}$ with quadrupolar excitation at $3.0 \mathrm{~T}[37,39]$ anticipate the results reported here. After incorporating the RF-only mode into the 3-T system and replacing quadrupolar excitation, we obtained the spectra shown in Figure 3 of 

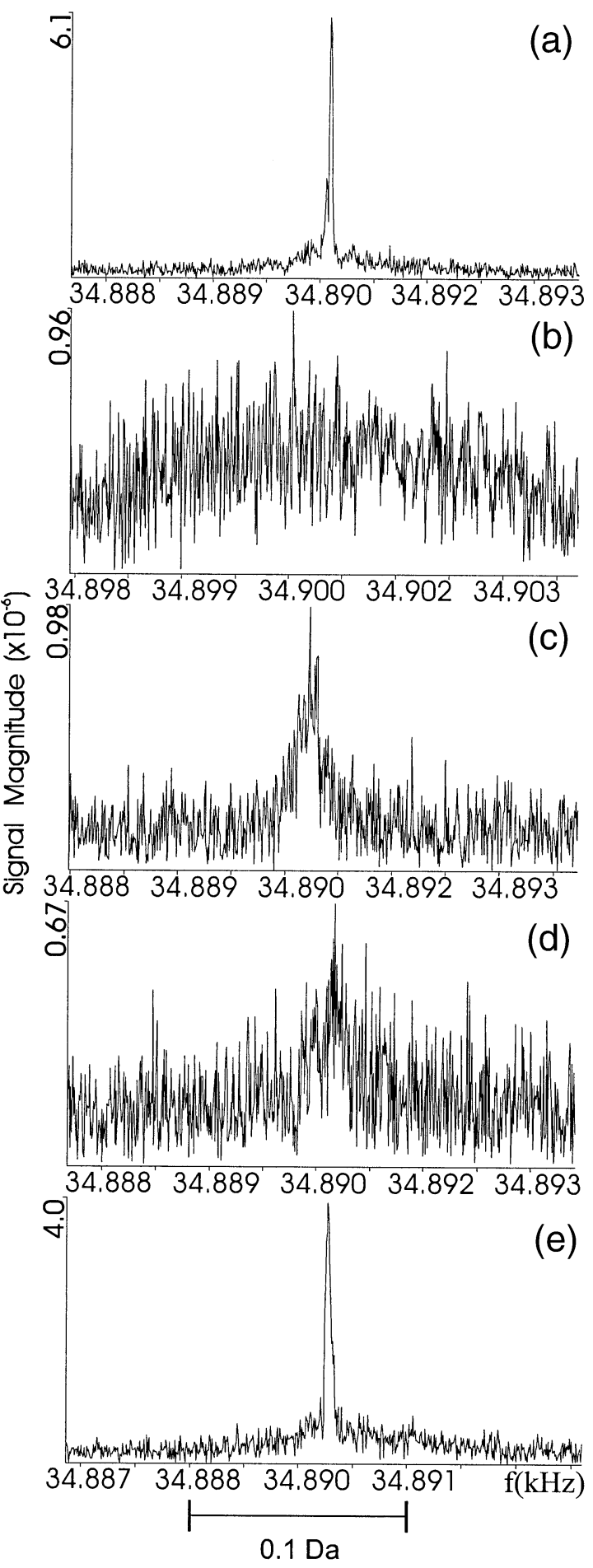

Figure 3. In-field MALDI of substance $P(34,890.5 \mathrm{~Hz})$. (a) Sequence includes a shaped retarding field, a low-mass-eject event, an RF-only-mode event and compensation (RP $=850 \mathrm{~K})$. (b) Compensation turned off. (c) RF-only-mode event disabled (RP = $170 \mathrm{~K})$. (d) Low-mass eject disabled. (e) Shaped retarding potential replaced with conventional one, $0 \mathrm{~V}$ to $10 \mathrm{~V}(\mathrm{RP}=450 \mathrm{~K})$.

the $[\mathrm{M}+\mathrm{H}]^{+}$ion of substance $\mathrm{P}(\mathrm{m} / \mathrm{z}=1348.6)$ generated by in-field MALDI under various conditions. Figure 3 a shows a peak shape obtained with a sequence that includes a shaped retarding field, a low-mass-eject event, an RF-only-mode event, and compensation; this panel serves as a reference point for comparison with the other peak shapes in the figure. The shaped retarding field employed here is different than that used by Wilkins and co-workers [6] and is discussed more fully in the experimental section.

The full combination provides a compelling advantage. Compensating the electric fields in the vicinity of the detection cyclotron orbits [frequency focusing (42, 43)] vastly improves peak shape: compare Figure $3 b$, where the peak from an uncompensated trap was too wide to fit into the window $(\sim 20 \mathrm{~K} \mathrm{RP})$, to Figure $3 \mathrm{a}$, where the peak from a compensated trap produced a resolving power (RP) of $860 \mathrm{~K}$. The use of the RF-onlymode would be expected to pressure focus the cloud and consequently reduce the range of field inhomogeneities sampled by the detected cyclotroning ions. The reduction in the sampled field inhomogeneities, apparently, is not sufficient to provide a substantial improvement in resolving power for the uncompensated trap (compare Figures $3 a$ and $3 b$ ). One explanation is found in frequency vs. initial-mode-amplitude plots, which show that in an uncompensated trap, broad peaks are produced because frequency varies considerably for even a moderate range of $z$-mode amplitudes. In the compensated trap, however, frequencies are at a saddle point, and sharp peaks are produced because frequency is nearly constant for a moderate range of $\mathrm{z}$-mode amplitudes. The peak shape in Figure 3a is non Lorentzian possibly because the compensation has revealed higher order field imperfections.

Using the RF-only mode to reduce the ion-cloud size also improves peak shape and sensitivity: compare Figure 3c, where the RF-only mode had been removed, causing the RP to decrease to $170 \mathrm{~K}$, to Figure $3 \mathrm{a}$. Pressure focusing of the ion cloud with the RF-onlymode event [36] allows us to fit mode-amplitude distributions into the constant frequency range of the compensated trap.

For comparison, Easterling et al. [44] obtained gramicidin a resolving power of $1.5 \mathrm{M}$ for gramicidin $\mathrm{S}$ ions $(\mathrm{m} / \mathrm{z}=1163.7)$ desorbed in field of a $4.7-\mathrm{T}$ instrument with a Nd:YAG laser (355 nm) and axialized with quadrupolar excitation. Allowing for the higher magnetic induction and the slightly lower mass, we translate the $860-\mathrm{K}$ resolving power shown in Figure 3a (as $\left.\mathrm{B}^{2} /(\mathrm{m} / \mathrm{z})\right)$ to $2.4 \mathrm{M}$ for the Easterling experiment.

We believe that coulombic forces from the unexcited matrix ions that remain at the origin of the trap degrade performance: compare Figure $3 \mathrm{~d}$, where a low mass ejection chirp ( $\mathrm{m} / \mathrm{z}$ up to 500) was turned off, causing a reduction in RP and signal-to-noise ratio, to Figure $3 \mathrm{a}$. Further proof for the attribution to unexcited matrix ions, or even to fragment ions, must be the subject of future work. Gated trapping with a shaped retarding field improves ion catching efficiency over a more conventional retarding field, which produced a $450-\mathrm{K}$ $\mathrm{RP}$ peak (compare Figure $3 \mathrm{e}$ to $3 \mathrm{a}$ ). When we did not 

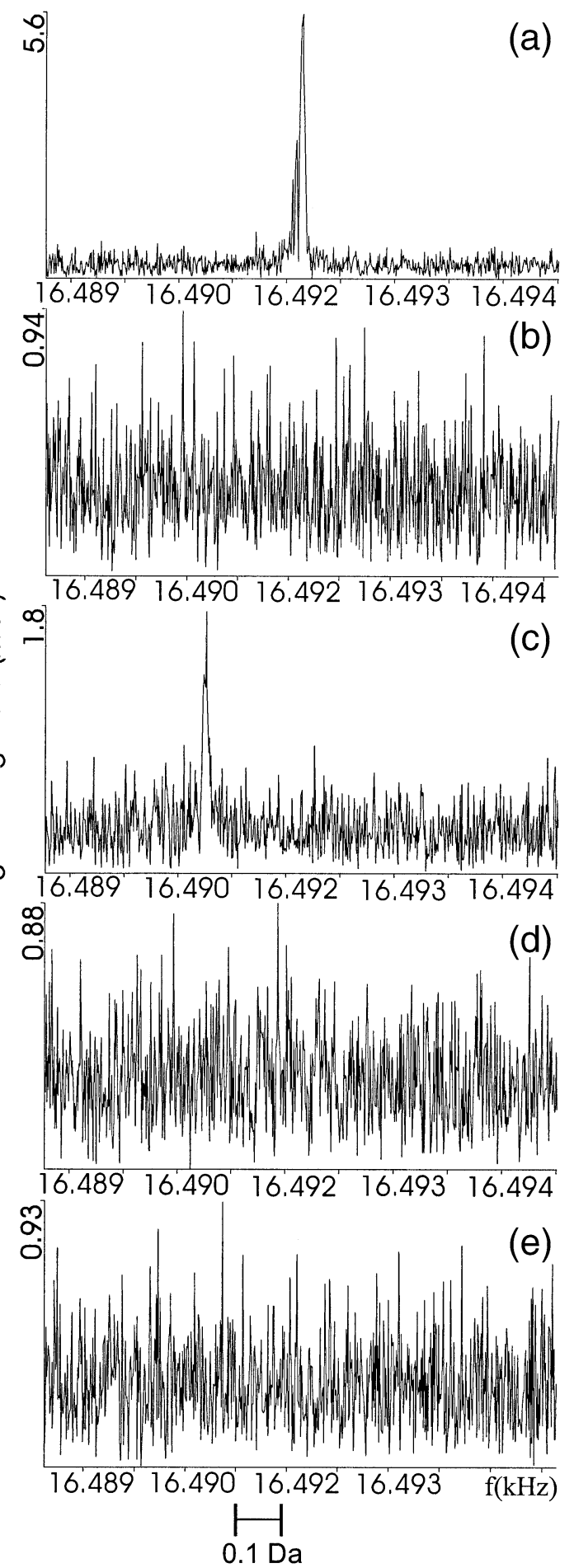

Figure 4. In-field MALDI of Melittin $(16,494 \mathrm{~Hz})$. (a) Sequence includes a shaped retarding field, a low-mass-eject event, an RF-only-mode event and compensation $(\mathrm{RP}=278 \mathrm{~K})$. (b) Compensation turned off. (c) RF-only-mode event disabled ( $R P=234$ K). (d) Low-mass ejection range pulled back from $\mathrm{m} / \mathrm{z} 2353$ to $\mathrm{m} / \mathrm{z}$ 523. (e) Same as (d) except RF-only-mode was disabled.

use the RF-only mode and compensation, we found no signals.

Do these advantages apply for higher $m / z$ ions? At roughly double the mass, the combination of gated trapping, low-mass eject, RF-only mode, and trap compensation allows the in-field MALDI-generated [M + $\mathrm{H}]^{+}$ion of melittin $(\mathrm{m} / \mathrm{z} 2845.7)$ to be detected (Figure $4 a)$. A comparison of Figure $4 a$ with $4 \mathrm{~b}$ shows that ions in an uncompensated trap produce no detectable signal. If we replace the RF-only mode with a 0.1-s pressure pulse, we see (compare Figure $4 \mathrm{a}$ with $4 \mathrm{c}$ ) a signal that is reduced in amplitude (by 3 times) and resolving power $(280 \mathrm{~K}$ to $230 \mathrm{~K})$. When we turned off the He pulse used to obtain Figure $4 \mathrm{c}$ and made no other modification to the sequence, we detected no signal. We again found that the omission of a low-mass eject (up to $m / z$ 2353) gave no detectable signal, suggesting that coulombic forces from the unexcited matrix ions that remain at the origin of the trap degrade performance: compare Figure $4 \mathrm{~d}$ and $4 \mathrm{e}$ with $4 \mathrm{a}$. In an effort to reduce the overall time of the experiment, we carefully optimized the time for the RF-only-mode-event and found that we could achieve the same result with a much shorter time. The RF-only mode event time was longer in the experiments with substance P. We suggest that the longer RF-only mode event produces a tighter ion cloud, as evidenced by a shift to lower frequency and lower resolving power (data not shown).

In conclusion, we have evidence that the problems of detecting MALDI-produced ions (low frequency, relatively high $m / z$ ) in FTMS can be overcome by a judicious combination of ion injection, ion-cloud size reduction, and trap compensation. We suggest that this demonstration for in-field MALDI has broader implications for the detection by FTMS of high $\mathrm{m} / \mathrm{z}$ ions formed by any means.

\section{Acknowledgment}

This research was supported by the National Center for Research Resources of the NIH (Grant No. P41RR00954).

\section{References}

1. Gluckmann, M.; Karas, M. The initial ion velocity and its dependence on matrix, analyte and preparation method in ultraviolet matrix-assisted laser desorption/ionization. J Mass Spec 1999, 34, 467-477.

2. Koster, C.; Castoro, J. A.; Wilkins, C. L. High-resolution matrix-assisted laser desorption/ionization of biomolecules by Fourier transform mass spectrometry. J. Am. Chem. Soc 1992, 114, 7572-7574.

3. Solouki, T.; Russell, D. H. Laser desorption studies of high mass biomolecules in Fourier-transform ion cyclotron resonance mass spectrometry. Proc. Natl. Acad. Sci. U. S. A. 1992, 89, 5701-5704.

4. Baykut, G.; Jertz, R.; Witt, M. Bruker Daltonik GmbH, B. G. Matrix-assisted laser desorption/ionization Fourier transform ion cyclotron resonance mass spectrometry with pulsed insource collision gas and in-source ion accumulation. Rapid Commun. Mass Spectrom 2000, 14, 1238-1247.

5. McIver, J., Robert; Li, Y.; Hunter, R. L. High-resolution laser desorption mass spectrometry of peptides and small proteins. Proc. Natl. Acad. Sci. U. S. A. 1994, 91, 4801-4805. 
6. Yao, J.; Dey, M.; Pastor, S. J.; Wilkins, C. L. Analysis of High-Mass Biomolecules Using Electrostatic Fields and Matrix-Assisted Laser Desorption/Ionization in a Fourier Transform Mass Spectrometer. Anal. Chem 1995, 67, 3638-3642.

7. Easterling, M. L.; Mize, T. H.; Amster, I. J. MALDI FTMS analysis of polymers: improved performance using an open ended cylindrical analyzer cell. Int. J. Mass Spectrom. Ion Proc 1997, 169/170, 387-400.

8. Knobeler, M.; Wanczek, K. P. Theoretical investigation of improved ion trapping in matrix-assisted laser desorption/ ionization Fourier transform ion cyclotron resonance mass spectrometry: independence of ion initial velocity. Int. J. Mass Spectrom. Ion Proc 1997, 163, 47-68.

9. Hendricson, C. L.; Drader, J. J.; Quinn, J. P.; Mize, T. H.; Amster, J.; Marshall, A. G. Design and Performance of an 11-Tesla MALDI FT-ICR Mass Spectrometer, Proceedings of the 47th ASMS Conference on Mass Spectrometry and Allied Topics, Dallas, Texas, June 13-17, 1999.

10. Rempel, D. L.; Gross, M. L. A gated trapping strategy with a two-time constant and a delay for catching in-field generated ions that range over three decades in mass-to-charge and two decades in velocity in fourier-transform mass spectrometry. J. Am. Soc. Mass Spectrom 2001, 12, 296-303.

11. Frankevich, V.; Zenobi, R. Deceleration of high-energy matrixassisted laser desorption/ionization ions in an open cell for fourier transform ion cyclotron resonance mass spectrometry. Rapid Commun. Mass Spectrom 2001, 15, 2035-2040.

12. Van Dyck, R. S., Jr.; Schwinberg, P. B. Preliminary proton/ electron mass ratio using a compensated quadring Penning trap. Physical Review Letters 1981, 47, 395-398.

13. Van Dyck, R. S., Jr.; Wineland, D. J.; Ekstrom, P. A.; Dehmelt, H. G. High mass resolution with a new variable anharmonicity Penning trap. Applied Physics Letters 1976, 28, 446-448.

14. Gabrielse, G.; MacKintosh, F. C. Cylindrical Penning traps with orthogonalized anharmonicity compensation. Intl. J Spec Ion Proc 1984, 57, 1-17.

15. Gabrielse, G. Relaxation calculation of the electrostatic properties of compensated Penning traps with hyperbolic electrodes. Physical Review A: Atomic, Molecular, and Optical Physics 1983, 27, 2277-2290.

16. Naito, Y.; Inoue, M. Improvement of the electric field in FT-ICR trapped ion cell, Presented at the 36th Conference on Mass Spectrometry and Allied Topics, San Francisco, CA, June 5-10, 1988, 608-609.

17. Naito, Y.; Fujiwara, M.; Inoue, M. Improvement of the electric field in the cylindrical trapped-ion cell. Int. J. Mass Spectrom. Ion Proc 1992, 120, 179-9218.

18. Jackson, G. S.; White, F. M.; Guan, S.; Marshall, A. G. Matrix-shimmed ion cyclotron resonance ion trap simultaneously optimized for excitation, detection, quadrupolar axialization, and trapping. J. Am. Soc. Mass Spectrom. 1999, 10, 759-769.

19. Schweikhard, L.; Becker, S.; Bollen, G.; Kluge, H. -J.; Savard, G.; Stolzenberg, H.; Wiess, U.; Moore, R. B. A new cooling mechanism for heavy ions in a penning trap, Presented at the 38th Conference on Mass Spectrometry and Allied Topic, Tucson, AZ, June 3-8, 1990, 415-416.

20. Savard, G.; Becker, S.; Bollen, G.; Kluge, H. J.; Moore, R. B.; Otto, T.; Schweikhrad, L.; Stolzenberg, H.; Wiess, U. A new cooling technique for heavy ions in a Penning trap. Physics Letters A 1991, 158, 247-252.

21. Schweikhard, L.; Guan, S.; Marshall, A. G. Quadrupolar excitation and collisional cooling for axialization and high pressure trapping of ions in Fourier transform ion cyclotron resonance mass spectrometry. Int. J. Mass Spectrom. Ion Proc 1992, 120, 71-83.

22. Guan, S.; Kim, H. S.; Marshall, A. G.; Wahl, M. C.; Wood, T. D.;
Xiang, X. Shrink-wrapping an ion cloud for high-performance Fourier transform ion cyclotron resonance mass spectrometry. Chem. Rev. (Washington, D. C.) 1994, 94, 2161-2182.

23. Fisher, E. Die dreidiemensionale stabilisierung von landungstragern in einem vierpolfeld (Three-dimensional stabilization of charge carriers in a quadrupole field). Z. Phys. 1959, 156, 1-26.

24. Li, G. A quantum particle in a combined trap. Zeitschrift fuer Physik D: Atoms, Molecules and Clusters 1988, 10, 451-456.

25. Li, G. Z.; Werth, G. The combined trap and some possible applications. Physica Scripta 1992, 46, 587-592.

26. Huang, Y.; Li, G.-Z.; Guan, S.; Marshall, A. G. A combined linear ion trap for mass spectrometry. J. Am. Soc. Mass Spectrom 1997, 8, 962-969.

27. Gorshkov, M. V.; Guan, S.; Marshall, A. G. Dynamic ion trapping for Fourier-transform ion cyclotron resonance mass spectrometry: simultaneous positive- and negative-ion detection. Rapid Commun. Mass Spectrom 1992, 6, 166-172.

28. Rempel, D. L. Improved peak shapes from a modified FTMS cubic cell incorporating segmented trap plates, Presented at the 35th Conference on Mass Spectrometry and Allied Topics, Denver, CO, May 24-29, 1987, pp. 1124-1125.

29. Yang, S. S.; Rempel, D. L.; Gross, M. L. Exact mass errors in fourier transform mass spectrometry: experimental evidence for indirect coulombic mechanisms, Presented at the 36th Conference on Mass Spectrometry and Allied Topics, San Francisco, CA, June 5-10, 1988, pp. 586-587.

30. Yang, S. S.; Rempel, D. L.; Gross, M. L. A model for the reduction of mass measurement errors due to ion-ion interaction in FTMS, Presented at the 37th Conference on Mass Spectrometry and Allied Topics, Miami Beach, FL, May 21-26, 1989, 1224-1225.

31. Rempel, D. L.; Grese, R. P.; Gross, M. L. A scaling technique for studying the dynamics of high mass ions in Fourier transform mass spectrometry: a preliminary report. Int. J. Mass Spectrom. Ion Proc 1990, 100, 381-395.

32. Gross, M. L.; Cerny, R. L.; Giblin, D. E.; Rempel, D. L.; MacMillan, D. K.; Hu, P.; Holliman, C. Mass spectrometric methods: an answer for macromolecule analysis in the 1990s. Anal. Chim. Acta 1991, 250, 105-130.

33. Rempel, D. L.; Gross, M. L. Ion "optics" in FTMS and a RF-only mode event for the FTMS sequence as an example, Presented at the 38th Conference on Mass Spectrometry and Allied Topics, Tucson, AZ, June 3-8, 1990, pp. 884-885.

34. Rempel, D. L.; Gross, M. L. High pressure trapping in Fourier transform mass spectrometry: a radiofrequency-only-mode event. J. Am. Soc. Mass Spectrom 1992, 3, 590-594.

35. Rempel, D. L.; Gross, M. L. Three dimensional RF-only-mode stability diagrams in FTMS, Presented at the 46th Conference on Mass Spectrometry and Allied Topics, Orlando, FL, May 31-June 4, 1998, p 512.

36. Jacoby, C. B.; Holliman, C. L.; Rempel, D. L.; Gross, M. L. Ion cloud manipulation using the radiofrequency-only-mode as an improvement for high mass detection in Fourier-transform mass spectrometry. J. Am. Soc. Mass Spectrom 1993, 4, 186-189.

37. Holliman, C. L.; Rempel, D. L.; Gross, M. L. Detection of high mass-to-charge ions by Fourier transform mass spectrometry. Mass Spectrom. Rev 1994, 13, 105-132.

38. Gooden, J. K.; Rohrs, H.; Russell, A.; Rempel, D.; Gross, M. L. Improved performance using a compensated cubic trap for FTMS studies of MALDI generated ions, Presented at the 47th Conference on Mass Spectrometry and Allied Topics, Dallas, TX, June 13-17, 1999.

39. Rohrs, H. W.; Gooden, J. K.; Rempel, D. L.; Russell, A. L.; Gross, M. L. Ion-Focusing Events in FTMS Studies of MALDIDerived Ions, Presented at the 47th Conference on Mass Spectrometry and Allied Topics, Dallas, TX, June 13-17, 1999. 40. Rempel, D. L.; Gooden, J. K.; Russell, A. L.; Barbacci, D. C.; 
Gross, M. L. RF-Only Mode, Frequency Focusing and Gated Trapping for In-Field MALDI FTMS: Operation Considerations, Presented at the 50th Conference on Mass Spectrometry and Allied Topics, Orlando, FL June 2-6, 2002.

41. Rempel, D. L.; Gross, M. L. A radio/trap supply design that accommodates both trap electric field compensation and rapidly switched gated trapping for in-field MALDI fourier transform mass spectrometry, Presented at the 48th Conference on Mass Spectrometry and Allied Topics, Long Beach, CA, June 11-15, 2000.

42. Rempel, D. L.; Holliman, C. L.; Gross, M. L. A theoretical treatment of first order triple frequency focusing for the FTMS cubic trap, Presented at the 42th Conference on Mass Spectrometry and Allied Topics, Chicago, IL, May 29-June 3, 1994, p 727.

43. Rempel, D. L.; Gross, M. L. Examples in hierarchies of optimally triple frequency focused FTMS cubic traps, Presented at the 43th Conference on Mass Spectrometry and Allied Topics, Atlanta, GA, May 21-26, 1995, p 786.

44. Easterling, M. L.; Pitsenberger, C. C.; Amster, I. J. RF capacitive coupling with efficient gated trapping in internal matrix-assisted laser desorption ionization Fourier transform ion cyclotron resonance. J. Am. Soc. Mass Spectrom 1997, 8, 195-198. 